\title{
I.S.U.S. PROJECT (ITALIAN SKIN ULCER STUDY) (1 January 2015-31 December 2016). Final data
}

Francesco Petrella, Franca Abbritti, Francesca Bachetti, Sonia Brizzi, Michele Bruscella, Piero Baù, Mario Cala, Ferdinando Campitiello, Giovanna Capace, Massimo Calveri, Marina Comandini, Giovanni Vito Corona, Teresa Cosentino, Amarilli Croce Casalena, Cinzia Datteri, Cristiana Di Campli, Raffaele Di Nardo, Enrica Di Spirito, Filomena Discenza Spensieri, Ciro Falasconi, Michele Angelo Farina, Caterina Favaro, Domitilla Foghetti, Andrea Frasca, Manuela Galleazzi, Pierluigi Gallo, Luca Gazzabin, Ivo Gerbelle, Manuela Germano, Francesco Giacinto, Serena Giannini, Giorgio Guarnera, Klarida Hoxha, Vincenzo Labbro, Vincenzo Lauletta, Deianira Luciani, Giuseppe Maierà, Giuseppe Maccarone, Cosimo Maglio, Paolo Mascioli, Vincenzo Mattaliano, Massimo Menculini, Piermauro Miraglia, Giovanni Battista Mosti, Marcello Napolitano, Giuseppe Nebbioso, Pamela Orazi, Fabio Pacifico, Maurizio Palombi, Piergiorgio Pastore, Marina Pierangeli, Stefano Priolo, Marco Romanelli, Donatella Rossolini, Elisa Ronzullo, Maria Teresa Scalise, Patrizia Scarpelli, Piero Secreto, Claudio Solinas, Marilena Tender, Stefano Trovò

Italian Association of Skin Ulcers, Turin, Italy

\section{PREMISE}

The Italian National Healthcare System (NHS) processes data concerning outpatient services at public facilities based exclusively upon the weight of services provided. It is the Ministry of Health which initially determines the types of services that can be provided for each pathology. In Italy today (as we await the publication of the new Essential Care Levels already identified by the Ministry of Health) there are still no medical codes specifically associated with specialist outpatient services for the treatment of skin ulcers. This means that not all patients affected by this pathology who turn to public facilities are traceable. It is different where hospital admission is concerned because data is usually obtained from the discharge forms. Unfortunately, the data obtained is not always a true expression of the prevalence of skin ulcers in the hospital environment. In many cases the prevalence of pressure ulcers is not properly reported. This is because their appearance during hospitalization is an indicator of poor hospital care. The lack of data makes it impossible for our NHS to plan appropriate interventions in this area, both organizationally and economically. Scarce financial resources are allocated for the treatment of skin ulcers and their costs fall almost entirely upon the patients and their families. Furthermore, there are no national care networks dedicated specifically to their treatment.

\section{OBJECTIVE}

The objective of this study is to directly collect the largest possible amount of data on patients affected by skin ulcers in Italy.

Correspondence: Italian Association of Skin Ulcers, Via San Francesco da Paola 37, 10123 Turin, Italy. Tel: +39.011.2446911 - Fax: +39.011.2446950

E-mail: info@aiuc.it

Key words: Italian Skin Ulcer Study; Italian Association of Skin Ulcers; Italian National Healthcare System.

Received for publication: 31 December 2016.

Accepted for publication: 28 February 2017.

This work is licensed under a Creative Commons Attribution NonCommercial 4.0 License (CC BY-NC 4.0).

CCopyright F. Petrella et al., 2017

Licensee PAGEPress, Italy

Italian Journal of Wound Care 2017; 1(1):1-8

doi:10.4081/ijwc.2017.1

\section{METHOD}

The method adopted does not include any kind of filter and/or inclusion and/or exclusion criteria. The procedure used for data entry is very similar to the procedure for entering data into a patient's register. Additionally, the methodology adopted for the realization of this study complies with the current laws on personal data protection.

The parameters to be evaluated were established beforehand and are all normally acquired by healthcare professionals during treatment. The data collected are:
1. Tax code
2. Year of birth
3. Province of residence
4. Gender
5. Etiology
6. Site (main ulcer)
7. Size (expressed in $\mathrm{cm}^{2}$ )
8. Number
9. Month of onset
10. Year of onset
11. Place of treatment 
12. Hospitalization (No/Yes)

13. Admission in protected structures $(\mathrm{No} / \mathrm{Yes})$

An electronic form with the parameters specified above and with multiple choice answers was created. A proprietary electronic platform to upload the data was created on the website www.aiuc.it. The data is collected anonymously. The only sensitive data required during the entry phase is the patient's tax code. This data is used only to verify the presence of the patient in the registry in order to avoid entering duplicate data which might distort the study. In actuality the system does not perform searches based on the actual tax code, but by comparing an SHA1 (Secure Hash Algorithm) with those previously stored. An SHA-1 produces a 160-bit encrypted string and is a so-called one-way, collision-free algorithm. Thanks to this particular technology it is possible to create a single encrypted version of each piece of data. This procedure makes it impossible for anyone to trace the original data (tax code) from the encrypted version.

Furthermore, it is impossible to have two equal strings for two different original codes. A positive match with the encrypted key generated by the entered tax code indicates that the patient has already been entered into the database. In this case, the insertion of duplicate patient data is blocked and reported to the operator.

To access the system it is necessary to request access credentials (username and password). The system generates them automatically after verifying that the applicant is an AIUC member. Only AIUC members can participate in the project. The adopted methodology was designed in full compliance with the provisions contained in resolution no. 85/2012 of the Italian Data Protection Authority.

The electronic platform automatically processes the data that was entered. It automatically generates a general report and reports on a regional basis. The report shows the data only in an aggregate form as shown in Table 1.

The project will last for two years, from 1 January 2015 to 31 December 2016.

Table 1.

Patients - gender - average age - number of lesions

Distribution of patients by healthcare facility type

Distribution of skin ulcers by etiology

Distribution of skin ulcers by anatomical site

Average skin ulcer area by etiology

Hospitalization

Admissions in protected healthcare structures

\section{RESULTS}

The data to follow is shown exclusively in an integrated form and was generated automatically by the system on 31 December 2016.

- Patients - gender - average age - number of lesions (Figure 1).

- Distribution of patients by healthcare facility type.

Data on healthcare settings (Figure 2).

- Distribution of skin ulcers by etiology.

The data was divided according to the etiological nature of the lesion (Figure 3).

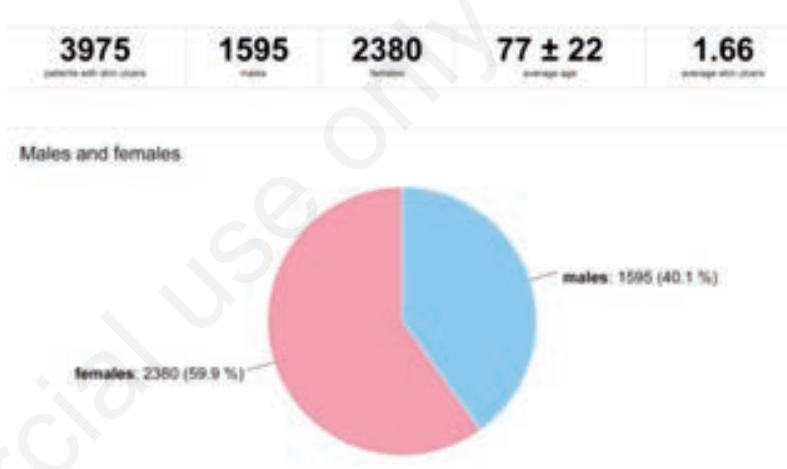

Figure 1.

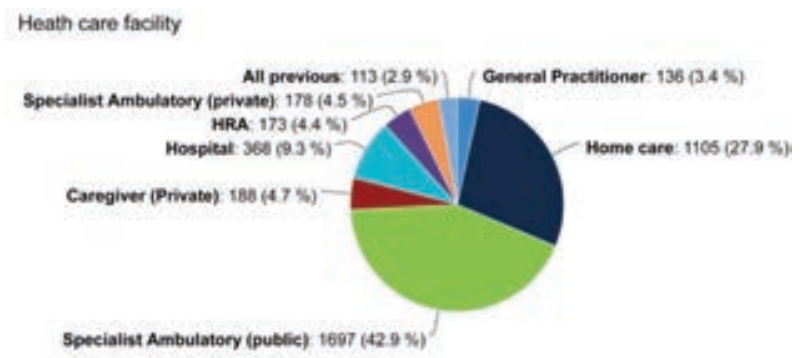

Figure 2.

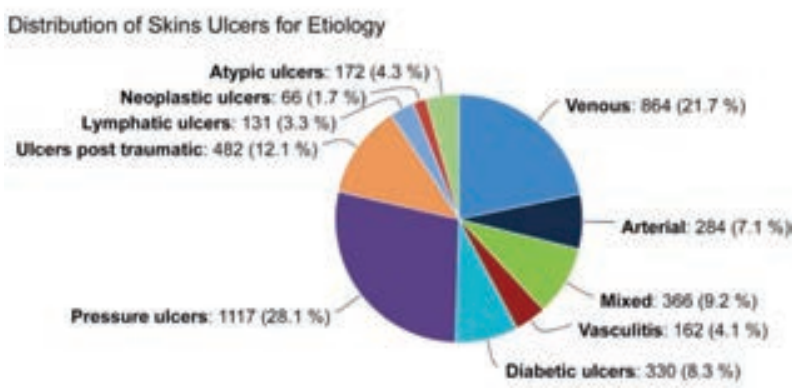

Figure 3. 
- Distribution of skin ulcers by anatomical site. Highlights by percentage the anatomical distribution of skin ulcers (Figure 4).

- Average skin ulcer area by etiology.

Data on the average size, expressed in $\mathrm{cm}^{2}$, classified on an etiological basis (Figure 5).

- Hospitalization.

Data on the number of patients suffering from skin ulcers who, during their clinical history, have resorted to hospitalization (Figure 6).

- Admissions in protected healthcare structures.

The data shows the number of patients with skin ulcers

Ulcers distribution for Anatomical Sites

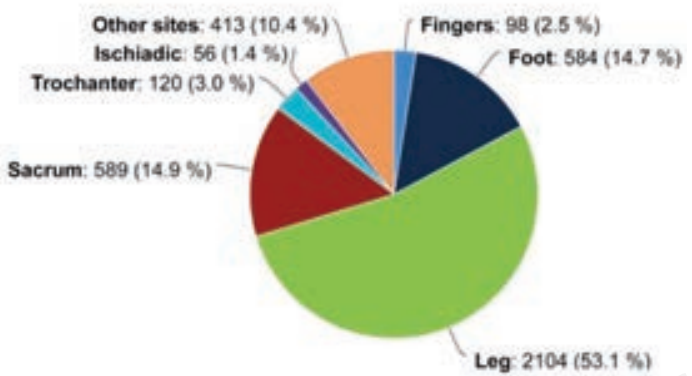

Figure 4.

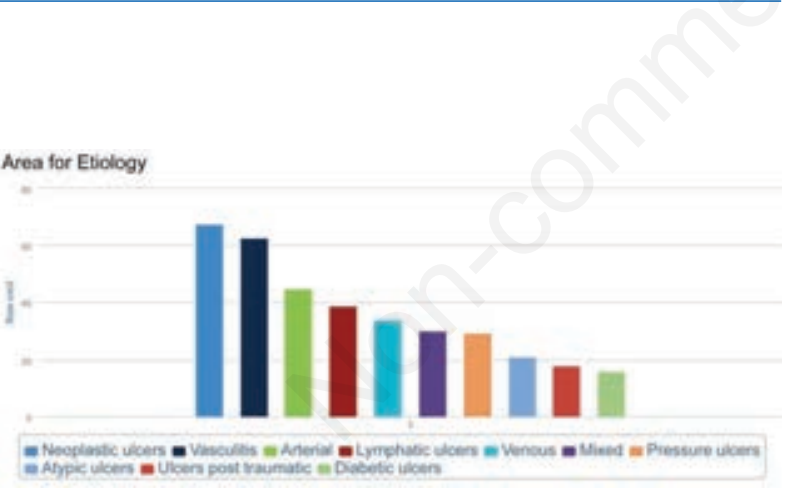

Figure 5.

Hospitalization

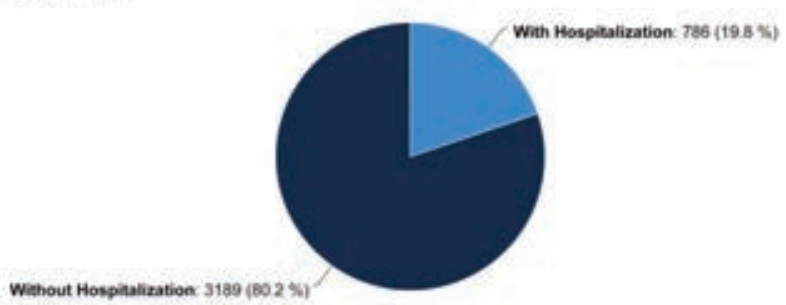

Figure 6. who were treated in protected healthcare structures (Figure 7).

- Healing time of skin ulcer.

Data on the time between the appearance of the lesion and the inclusion in the database (Figure 8).

\section{DISCUSSION}

The data included is affected by the patient's reporting location and by an eventual specialization of the healthcare center and/or of the specific healthcare settings. Therefore, an analysis of the data must always take into consideration the methodology that was adopted. The majority of patients appear to be female. The overall average age is 77 with 1.66 lesions per patient. This highlights how, in a high percentage of cases, the same patient suffers from multiple ulcerative lesions. Among enrolled patients, $80 \%$ turned to public facilities belonging to our NHS primarily to public specialist outpatient clinics and home care services (ADI).

A very interesting piece of data that emerges is the huge etiological variability of the lesions. This highlights an increased need to be able to make a correct and quick differential diagnosis. A need that is also supported by

\section{Admissions in Protected Structures}

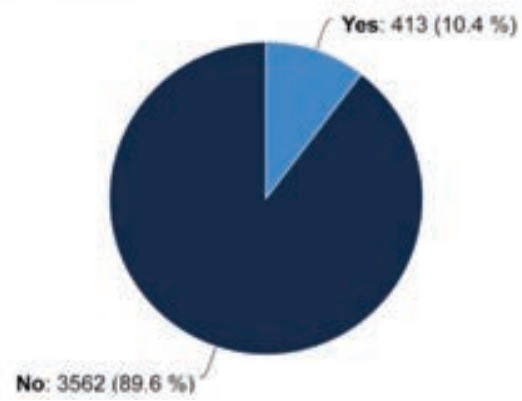

Figure 7.

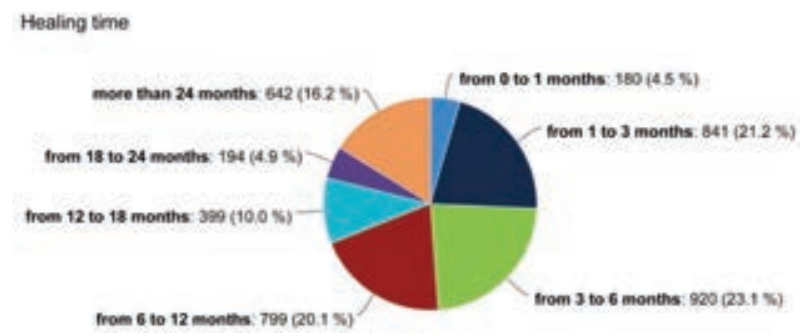

Figure 8. 
data on the prevalence of venous ulcers, which appears to be very low. Only $21.7 \%$. A figure that lends itself to two different interpretations: the first being that many venous lesions heal through simple therapeutic paths that do not require the intervention of specialists; the second being that in the past the percentage of venous ulcers had been overestimated (at the moment we are not able to provide data on its true incidence).

Specialist outpatient facilities are the setting of reference for ulcerative skin lesions with various etiologies for the self-sufficient patient. This figure is confirmed when one considers that only $9.3 \%$ of patients were found to be hospitalized during the project. Within the context of home care (ADI), the data between a healthcare setting (ADI 27.9\%) and etiology of the lesion (Pressure ulcers $28.1 \%$ ), are almost superimposable. Therefore, the $100 \%$ of patients who are bedridden or not self-sufficient suffer from pressure ulcers. If one compares the percentage of patients with lesions who resorted to admission in a protected structure $(10.4 \%)$ with those currently admitted to a protected structure (4.4\%), it should be noted that the patient is admitted to a protected structure for a limited amount of time. In view of these observations, the home turns out to be the main, if not the exclusive, healthcare setting for bedridden and/or non self-sufficient patients.

Further analysis of the data confirms that the main sites of ulcerative skin lesions are the lower limbs. Though the figures regarding the relationship between the size of the lesions and etiology seem to provide a single piece of information there is no direct relationship between etiology and lesion size at the moment. Lesion size seems almost exclusively determined by the severity of the primary pathology. More than 1235 patients out of the 3975 patients enrolled have suffered from skin ulcers for more than a year; of these, 642 patients for over two years. The data concerning the duration of ulcerative lesions confirms the difficulties patients encounter in locating competent healthcare facilities for the treatment of skin ulcers. While $19.8 \%$ of patients with skin ulcers had to resort to hospitalization, an analysis of the data shows that one skin ulcer patient out of five will be hospitalized at some point during their medical history. Data concerning the duration of ulcerative lesions seems to confirm the difficulties patients encounter in locating competent healthcare facilities for the treatment of skin ulcers.

\section{CONCLUSIONS}

The study unequivocally highlights the ineffectiveness of the organizational procedures in the treatment of patients suffering from skin ulcers in our country. Organizational failings are a direct consequence of the exclusion of wound care from the Essential Assistance Levels of the Italian NHS. Besides not having dedicated care pathways, our NHS crystallizes existing organizational problems without allowing the NHS itself to acknowledge and correct them. In fact, an analysis of the results shows how the absence of a skin ulcer treatment network within our NHS creates organizational distortions that lead to a lengthening of healing times and, ultimately, to increased suffering by patients, considerable economic impact due to ineffective procedures and, most of all, to a constant recourse to hospitalization in a high percentage of cases. 